\title{
Swift charged particles in a degenerate electron gas: An estimation for the Barkas effect in stopping
}

\author{
I. $\operatorname{Nagy}^{1,2}$ and I. Aldazabal ${ }^{3,2}$ \\ ${ }^{1}$ Department of Theoretical Physics, Institute of Physics, \\ Budapest University of Technology and Economics, \\ H-1521 Budapest, Hungary \\ ${ }^{2}$ Donostia International Physics Center, P. Manuel de Lardizabal 4, \\ E-20018 San Sebastián, Spain \\ ${ }^{3}$ Centro de Física de Materiales (CSIC-UPV/EHU)-MPC, P. Manuel de Lardizabal 5, \\ E-20018 San Sebastián, Spain
}

(Dated: December 17, 2018)

\begin{abstract}
Applying a recently suggested new form [Phys. Rev. A 94, 042704 (2016)] for the stopping power in terms of scattering phase shifts, here we show analytically that an exact leading phase shift may contain that information which is completely enough to characterize the asymptotic charge-sign-independent Bethe term $\left(\propto Z_{1}^{2}\right)$ and a charge-sign-dependent Barkas term $\left(\propto Z_{1}^{3}\right)$ in the stopping force of a degenerate electron gas for fast projectiles with charge $Z_{1}$. Our analytic implementation is based on a Hulthén-type potential with velocity-dependent screening. The next term in an asymptotic expansion, the Bloch term $\left(\propto Z_{1}^{4}\right)$, measures the difference of the exact and first-order Born treatments with Coulomb potential. We found a reduced value for the Barkas term, in comparison with the conventional estimation which rests on the transport cross section.
\end{abstract}

PACS numbers: $34.50 . \mathrm{Bw}$ 


\section{INTRODUCTION}

The first-order Born approximation for the phase shifts or for the real scattering amplitude is a familiar and convenient approximation for handling scattering problems. It can be adequate, or informative in so many cases that one tends to develop the habit of using it without always checking the conditions for its applicability [1]. However, when one considers the interaction of an electron via regularized potentials with swift attractive or repulsive charges moving in a degenerate charged fermion gas, the experimentally measurable quantity, the stopping power of this gas for projectiles, may show a deviation from the first-order estimation which results in the Bethe term $\left(\propto Z_{1}^{2}\right)$. The sign-dependent deviation from this leading term at high velocities is characterized by the next-to-leading term, i.e., the Barkas term $\left(\propto Z_{1}^{3}\right)$ in the stopping force. The existence of such a term in the stopping power of solids is well established experimentally, in particular at random-collision condition [2, 3] involving swift protons and antiprotons $\left(Z_{1}= \pm 1\right)$. The next higher-order term to the Bethe and Barkas terms in stopping is the so-called Bloch term $\left(\propto Z_{1}^{4}\right)$.

The magnitude and interplay of the Barkas and Bloch terms in modulating the leading Bethe term gave the background to dedicated early experiments [4] with bare intruders with atomic number $9 \leq Z_{1} \leq 17$ moving at a high velocity $\left(v \simeq 11 v_{0}\right)$ in channeling direction of a solid. From data-analysis it was concluded, using Lindhard's asymptotic theory [5], that either the higher-order terms, i.e., the Barkas and Bloch, are small to detect them or both may be large but virtually canceling each other out. However, Peierls' remark [1] is relavant to [4], since the Sommerfeld parameter $\gamma=Z_{1} e^{2} /(\hbar v)$ is not small. In fact, it is in the range of unity. Besides, Lindhard clearly emphasized in his work: I will try to show that the theory is quite simple basically, but this does not mean that I am sure of all details of it.

Notice at this point, that a more recent mean-field calculation [6], performed in the auxiliary orbital representation of time-dependent density-functional theory with $Z_{1}= \pm 1$ and $\left(v / v_{0}\right) \in(0,6)$ for metallic clusters, results in a very small Barkas effect already for about $v>3 v_{0}$. The above, experimental and theoretical, facts give the motivations to our contribution. We implement, analytically, a recently proposed form [7] for the stopping power which is also given in terms of scattering phase shifts. That form was termed as superior to the one used in [5]. Moreover, based on a numerical implementation, its possible relevance to understanding the charge-sign-dependent term was explicitly stressed [7]. 
This paper is organized as follows. The next Section is devoted to the theory and the discussion of the results obtained. The last Section contains the summary, and our comments. We will use natural (rather than Hartree atomic, where $e^{2}=\hbar=m=1$ ) units in this work, except where (in an illustrative Figure) the opposite is explicitly stated.

\section{THEORY AND RESULTS}

In energy-loss experiments we add an external, heavy charged projectile to the manybody system of interacting electrons. In a scattering description, the incoming one-electron states are plane waves due to the translational invariance of the target system with number density $n_{0}$. The momentum distribution of these states of real electrons is described by a renormalized distribution function. We are, therefore, at an important exceptional case where Landau's description for quasiparticle energies with a noninteracting-like Fermi-Dirac distribution function is not applicable [8]. Similarly, the simpler Kohn-Sham-like mean-field modeling with an ideal (step-function) distribution function to consider an averaging over momentum distribution, is incomplete. This problem is not investigated yet at low [9, 10]

and intermediate [11] velocities of charged projectiles. Peliminary results for the case of a fixed charged impurity are available in [12]. However, at high projectile velocities $(v)$, the relative velocity $\left(v_{r}\right)$ to the underlying two-body kinematics in stopping is given by

$$
v_{r}=v\left[1+\frac{1}{3} \frac{\left\langle v_{e}^{2}\right\rangle}{v^{2}}\right]
$$

where $v_{e}$ is the electron velocity [13]. In an interacting system $v_{e} \in(0, \infty)$, even at zero temperature. But, in spite of $\left\langle v_{e}^{2}\right\rangle>\left[(3 / 5)\left(3 \pi^{2} \hbar^{3} n_{0} / m\right)^{2 / 3}\right]$, at high velocities we can take, as expected, $v_{r}=v$. Thus, the wave number of an electron becomes $k=m v / \hbar$.

In order to motivate, heuristically as a first step, the basic expression used in this work for asymptotic stopping power calculation, we start by the Coulomb-potential case. Taking the Coulomb phase shifts [14] in a partial-wave expansion, we can write

$$
\sum_{l=0}^{\infty}(l+1) \sin ^{2}\left[\sigma_{l}(k)-\sigma_{l+1}(k)\right] \equiv \gamma \sum_{l=0}^{\infty} \sin \left[\sigma_{l}(k)-\sigma_{l+1}(k)\right] \cos \left[\sigma_{l}(k)-\sigma_{l+1}(k)\right]
$$

where $\gamma=Z_{1} e^{2} / \hbar v=Z_{1} /\left(a_{0} k\right)$, is the Sommerfeld parameter. The common Coulomb logarithm [14] does not appear in the differences $\left[\sigma_{l}(k)-\sigma_{l+1}(k)\right]=\arctan [\gamma /(l+1)]$. However, 
in the case of a screened (regularized) potential, $V(r)$, we get an inequality

$$
\sum_{l=0}^{\infty}(l+1) \sin ^{2}\left[\delta_{l}(k)-\delta_{l+1}(k)\right] \neq \gamma \sum_{l=0}^{\infty} \sin \left[\delta_{l}(k)-\delta_{l+1}(k)\right] \cos \left[\delta_{l}(k)-\delta_{l+1}(k)\right]
$$

where the Bessel phase shifts are denoted, as ususal, by $\delta_{l}(k)$. With a screened potential both sides of this inequality are finite and the left-hand-side is positive definite always. A truncated summation in the equality of Eq.(1), results in

$$
\sum_{l=0}^{L_{\max }}(l+1) \sin ^{2}\left[\sigma_{l}(k)-\sigma_{l+1}(k)\right]=\gamma^{2} \sum_{l=0}^{L_{\max }} \frac{l+1}{(l+1)^{2}+\gamma^{2}} .
$$

With $L_{\max } \rightarrow \infty$ one would get a divergency, as it is well-known with a three-dimensional Rutherford differential cross section to angle-weighting in the transport cross section.

Application of the left-hand-side of Eq.(2) is common [5, 10] in transport cross section calculations with spherical scattering potentials. The right-hand-side corresponds, precisely, to the new form proposed recently and considered as a superior form [7]. That proposal is based on calculating a noncentral induced electron density from which the stopping force is defined by integrating over the gradient of the corresponding (via convolution) electrostatic (Hartree) potential. This definition for the retarding force is classical in the sense that it does not reflect the probabilistic interpretation of quantum mechanics via complex matrix elements of a force operator. Indeed, the left-hand-side of Eq.(2) is expressible [15-19] as

$$
\sum_{l=0}^{\infty}(l+1)\left|i^{l}(-i)^{l+1} e^{i\left[\delta_{l}(k)-\delta_{l+1}(k)\right]}\left(\int_{0}^{\infty} d r r^{2} R_{l}(r) \frac{\partial U(r)}{\partial r} R_{l+1}(r)\right)\right|^{2},
$$

where, for simplicity, the $k$-dependence is not explicit in $R_{l}(k, r)$ which is a radial component in the partial-wave-based approach with spherical $U(r)=\left(2 m / \hbar^{2}\right) V(r)$. Contrary to this, the right-hand-side of Eq.(2) corresponds, formally, to the real part of complex elements

$$
\sum_{l=0}^{\infty}(l+1) \operatorname{Re}\left\{i^{l}(-i)^{l+1} e^{i\left[\delta_{l}(k)-\delta_{l+1}(k)\right]}\left[\int_{0}^{\infty} d r r^{2} R_{l}(r) \frac{2 m}{\hbar^{2}} \frac{\partial}{\partial r}\left(-\frac{Z_{1} e^{2}}{r}\right) R_{l+1}(r)\right]\right\},
$$

which has a simple mixed-product representation with two real terms as well

$$
\sum_{l=0}^{\infty}(l+1)\left(\int_{0}^{\infty} d r r^{2} R_{l}(r) \frac{\partial U(r)}{\partial r} R_{l+1}(r)\right)\left(\int_{0}^{\infty} d r r^{2} R_{l}(r) \frac{2 m}{\hbar^{2}} \frac{Z_{1} e^{2}}{r^{2}} R_{l+1}(r)\right) .
$$

The structure of this product shows that for swift projectiles, where the radial functions deviate only slightly from the corresponding plane-wave components, it will result in larger values than the square [15-19] of its first term with consistently employed $\partial U(r) / \partial r$. 
In this paper we will implement the right-hand-side of Eq.(2), and apply it at high velocities of projectiles to the recently proposed [7] stopping power expression

$$
\frac{d E}{d z}=\left(m v^{2}\right) n_{0} \frac{2 \pi}{k^{2}} \gamma \sum_{l=0}^{\infty} \sin \left\{2\left[\delta_{l}(k)-\delta_{l+1}(k)\right]\right\} .
$$

Since we focus on the $|\gamma|<1$ case in this work, we apply a two-term Taylor expansion

$$
\sum_{l=0}^{\infty} \sin \left\{2\left[\delta_{l}(k)-\delta_{l+1}(k)\right]\right\} \simeq 2 \delta_{0}(k)-\frac{4}{3} \sum_{l=0}^{\infty}\left[\delta_{l}(k)-\delta_{l+1}\right]^{3} .
$$

The dominant role of the leading $(l=0)$ phase shift is transparent in this asymptotic form. We proceed by using a Hulthén-type [20] effective scattering potential

$$
V(r)=-\frac{Z_{1} e^{2} \Lambda}{e^{\Lambda r}-1}
$$

with $Z_{1} \geq 1$ for attractive (bare) intruders. For antiprotons $Z_{1}=-1$. The screening parameter $\Lambda$ will be fixed below. For this potential, one has an exact solution for the Jost function, $F_{0}(k)$, of scattering theory [21 23]. The product representation for this function

$$
F_{0}(k)=\prod_{n=1}^{\infty}\left[1+\frac{i\left(Z_{1} / a_{0}\right)}{n(k-i n \Lambda / 2)}\right]
$$

allows the determination of the leading phase shift from $e^{2 i \delta_{0}(k)}=F_{0}(k) / F_{0}(-k)$. We get

$$
\delta_{0}(k)=\sum_{n=1}^{\infty} \arctan \left\{\frac{\gamma}{n\left[1-(\gamma \Lambda / 2 k)+(n \Lambda / 2 k)^{2}\right]}\right\} .
$$

This is an exact result. With $(\Lambda / 2 k) \ll 1$ in Eq.(6) one has

$$
\sum_{n=0}^{N_{\max }} \arctan \frac{\gamma}{n+1}=\sum_{l=0}^{L_{\max }}\left[\sigma_{l}(k)-\sigma_{l+1}(k)\right] .
$$

Therefore, an approximation [4] for $L_{\max }$ must respect the $\left(L_{\max } \Lambda / 2 k\right) \ll 1$ constraint.

Next, we determine the first-order Born result for $\delta_{l}^{B}(k)$ from

$$
\delta_{l}^{B}(k) \equiv-2 k \frac{m}{\hbar^{2}} \int_{0}^{\infty} d r r^{2} V(r) j_{l}^{2}(k r)=-\frac{m}{\hbar^{2}} \frac{1}{4 \pi} \frac{1}{k} \int_{0}^{2 k} d q q V(q) P_{l}(x),
$$

where $j_{l}(k r)$ is a spherical Bessel function of the first kind and $V(q)$ is the Fourier transform of a spherical potential $V(r)$. To the Legendre polynomials, we have $x=1-q^{2} /\left(2 k^{2}\right)$ as argumentum. In the Hulthén case, at $\Lambda \neq 0$, we can write

$$
V(r)=-Z_{1} e^{2} \Lambda \sum_{n=1}^{\infty} e^{-n \Lambda r}
$$




$$
V(q)=8 \pi Z_{1} e^{2} \Lambda^{2} \sum_{n=1}^{\infty} \frac{n}{\left[q^{2}+(n \Lambda)^{2}\right]^{2}}
$$

which make the calculation for the important $\delta_{0}^{B}(k)$ easy. In that case $P_{0}(u)=1$ in Eq.(7), and thus we obtain in first-order (plane-wave-based) Born approximation

$$
\delta_{0}^{B}(k)=\sum_{n=1}^{\infty} \frac{\gamma}{n\left[1+(n \Lambda / 2 k)^{2}\right]}=\frac{Z_{1}}{a_{0} k}[\operatorname{Re} \psi(1+i 2 k / \Lambda)-\psi(1)],
$$

in terms of standard digamma functions. In our asymptotic $(u \gg 1)$ case, we can apply the $[\operatorname{Re} \psi(1+i u)-\psi(1)]=(1 / 2) \ln \left(1+\Gamma^{2} u^{2}\right]$ approximation [24], where $\Gamma=1.781$. Comparison of Eq.(6) and Eq.(8) shows the fine details behind the applicability [1] of the first-order Born approximation. The phase shift must be small (i.e., $|\gamma|<1$ ) and, in addition, one has to neglect the charge-sign-sensitive $(\propto \gamma)$ term in the denominator of Eq.(6).

Now, let us go back to Eq.(2) and evaluate both sides with first-order Born approximation for the real scattering amplitude by considering the right-hand-side $(r h s)$ of Eq.(7) as well. From that evaluation, we get the following, very transparent, inequality

$$
\int_{0}^{2 k} d q q^{3}[V(q)]^{2}<\int_{0}^{2 k} d q q^{3}\left[\frac{4 \pi Z_{1} e^{2}}{q^{2}} V(q)\right] .
$$

The lhs, which rests on the conventional [5] transport cross section, tends more slowly (from below) to the Bethe limit than the rhs which is based on the new form in [7].

At this point, we return to the prescription of a reasonable screening parameter $\Lambda$. In order to follow as close as possible the motivating work in [7], where a Yukawa-type effective potential, $V(r)=\left(-Z_{1} e^{2} / r\right) \exp (-\lambda r)$, was used to numerics, we employ a scaling argument. Namely, we require the equality of the corresponding leading phase shifts at the Born limit. This constraint results in $\Lambda=1.781 \lambda$. Remarkably enough, this scaling is in a very nice agreement with an earlier [25] one $\Lambda=1.75 \lambda$. This latter is based on an entirely different, bound-state-related, physical problem. Following [26, 27] we take, as in the motivating [7], the $\lambda=\left(\omega_{p} / v\right)$ prescription, where $\omega_{p}=\left(4 \pi n_{0} e^{2} / m\right)^{1 / 2}$ is the classical plasma frequency.

With a two-term Taylor expansion, $\arctan (\alpha) \simeq\left[\alpha-(1 / 3) \alpha^{3}\right]$ in Eq.(6), and considering Eqs.(3-4) and Eqs.(7-8) as well, we reduce to the traditional [5] asymptotic form

$$
\frac{d E}{d z}=m\left(\omega_{p}^{2} a_{0}\right)\left(\frac{Z_{1}}{a_{0} k}\right)^{2}\left[L_{0}+Z_{1} L_{1}+Z_{1}^{2} L_{2}\right],
$$

with Bethe $\left(L_{0}\right)$, Barkas $\left(L_{1}\right)$, and Bloch $\left(L_{2}\right)$ terms, the following expressions

$$
L_{0}=\frac{1}{2} \ln \left[1+\left(\frac{2 k \Gamma}{\Lambda}\right)^{2}\right] \simeq \ln \frac{2 m v^{2}}{\hbar \omega_{p}},
$$




$$
\begin{aligned}
& L_{1}=\frac{\Lambda}{2 a_{0} k^{2}} L_{0} \simeq 0.89 \frac{\left(\omega_{p} / v\right)}{a_{0} k^{2}} \ln \frac{2 m v^{2}}{\hbar \omega_{p}}=0.89 \frac{e^{2} \omega_{p}}{m v^{3}} \ln \frac{2 m v^{2}}{\hbar \omega_{p}} \\
& L_{2}=-\frac{1}{3}\left(\frac{1}{a_{0} k}\right)^{2} \zeta(3)-\frac{2}{3}\left(\frac{1}{a_{0} k}\right)^{2} \zeta(3) \simeq-1.2\left(\frac{v_{0}}{v}\right)^{2} .
\end{aligned}
$$

In this equation the (1/3)-part comes from the second term of the above expansion for $\arctan (\alpha)$ in the leading phase shift, and the (2/3)-part rests on the second term in Eq.(4). Both terms are calculated for the $\Lambda \rightarrow 0$ case. This is an allowed approximation in the asymptotic limit since from Eq.(7) one can get easily (with a Yukawa-form) the estimation

$$
\left[\delta_{l}^{B}(k)-\delta_{l+1}^{B}(k)\right] \simeq \frac{\gamma}{(l+1)}\left[1-O\left(\lambda^{2} / k^{2}\right)\right] .
$$

Therefore, the Bloch term with screening tends from below to its Coulomb equivalent.

From the pioneering work [5] of Lindhard $(L)$ one has to the Barkas $\left(\propto Z_{1}^{3}\right)$ term

$$
L_{1}^{(L)}=\beta \frac{e^{2} \omega_{p}}{m v^{3}} L_{0}=\beta \frac{e^{2} \omega_{p}}{m v^{3}} \ln \frac{2 m v^{2}}{\hbar \omega_{p}},
$$

where $\beta=\pi$ or $\beta=(3 / 2) \pi$. One can see that this is essentially larger than ours in Eq.(11). Our result is in nice harmony with a previous one [6] obtained in a self-consistent, orbital-based mean-field approximation within TDDFT with cluster targets and $Z_{1}= \pm 1$ for projectiles. In that numerical modeling, the Barkas effect is also very small for $\left(v / v_{0}\right)>3$.

Lindhard's insightful estimation is based on a simple shifting, as $\left[k^{2}-\left(Z_{1} \beta \lambda / a_{0}\right)\right]$, in the scattering energy and using that shifted quantity in the pre-factor of a leading Bethe term. However, his leading term was calculated using the transport cross section, i.e., the lhs of the above inequality with real scattering amplitudes. It is easy to show, by taking a Yukawa-type modeling, that the mentioned inequality results in asymptotically

$$
\left(\ln \frac{2 m v^{2}}{\hbar \omega_{p}}-\frac{1}{2}\right)<\ln \frac{2 m v^{2}}{\hbar \omega_{p}} .
$$

We must note at this point, in favor of a late expert, that this lhs together with Lindhard's estimation for the Barkas term, would result in a reasonable numerical agreement with our present result, which is based on a different [7] modeling, for the case of proton $Z_{1}=1$ due to certain cancelation. But, in the case of an antiproton $Z_{1}=-1$, it would give a much stronger deviation from the Bethe limit.

After the above detailed analysis related to the stopping power of an electron gas for swift-projectiles, we continue with a quantitative comparison at high-velocities. We use to 


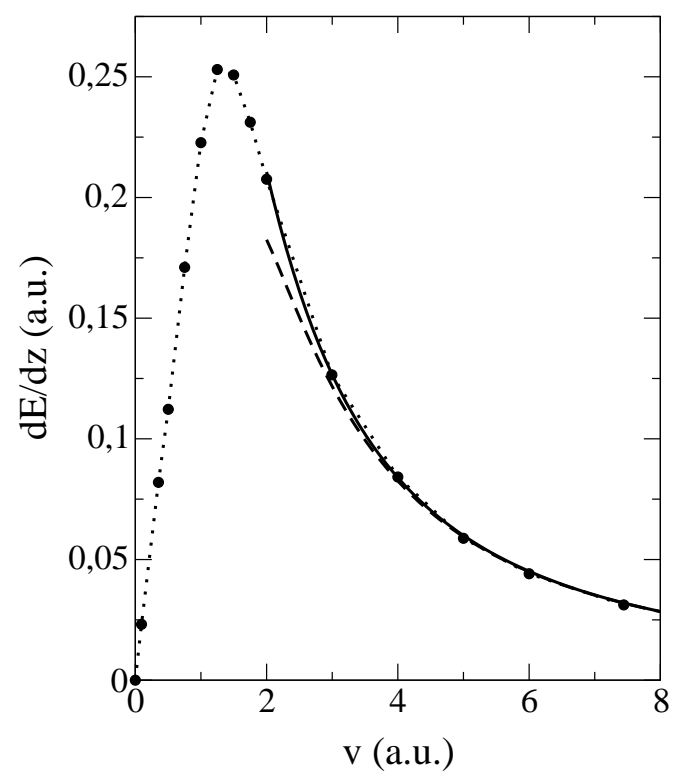

FIG. 1: Stopping powers, in atomic units, of an electron gas with $r_{s}=2.07$ for projectiles with $Z_{1}= \pm 1$. The solid $\left(Z_{1}=1\right)$ and dashed $\left(Z_{1}=-1\right)$ curves refer to the analytical approach detailed in this contribution. The numerics-based discrete dots (for $Z_{1}=1$ ) are taken from [28].

this comparison theoretical results from an independent source. Thus, in Fig.1, we exhibit our results for proton and antiproton, by solid and dashed curves, respectively. The discrete dots on the dotted curve are taken from [28] for $Z_{1}=1$. They are based on large-scale numerics [28] performed within TDDFT considering the valence part of a free-electron-like solid, Al. One can see a nice harmony between the solid curve and dots.

In a more general content, we should note that in the folklore of numerics [6, 28] it is customary to employ the first-principles and benchmark wording in order to emphasize a method-capability a priori. However, that wording is true only with a small but important modification. TDDFT, as well as the time-independent DFT, are first-principles approaches with semi-empirical inputs [29]. Thus they have an a posteriori character, as was emphasized in a pioneering work [9]. Comparisons with high-precision data or with exact [30] solutions on correlated models are always required in order to establish a transferable knowledge.

The two sides of the inequality, in Eq.(2), represent two approximations which give concordant results, mathematically, only for the $\Lambda=0$ case at which they yield a divergent expresssion for an observable quantity. The $l h s$ of Eq.(2) seems to be [11] the correct one at $v \rightarrow 0$, where the relative wave number is determined by the Fermi velocity. In that case the 
probabilistic interpretation of quantum mechanics, with squares of force matrix-elements, becomes transparent. Beyond that impurity limit and in a mean-field picture, which rests on projectile screening and scattering of independent electrons, a nonspherical multipole expansion [31] could be the consistent one. However, in such a treatment with nonspherical scattering potentials to determine the corresponding force-matrix elements [16], one arrives at coupled equations. A detailed investigation of this quantum-mechanical, probabilistic, approach is out of the scope of the present work and is left to a dedicated study.

The mathematical divergency of summations in Eq.(1) can be cured by taking a regularized (screened) two-body interaction. But that step can result in two different interpretations as well, as our diagnosis after Eq.(2) shows. We believe, therefore, that the two-dimensional version of the stopping problem [32, 33] may help in such interpretation-difficulties. There, with Coulomb interaction energy, we get to the corresponding transport cross section

$$
\sum_{m=0}^{\infty} \sin ^{2}\left[\sigma_{m}(k)-\sigma_{m+1}(k)\right] \equiv \sum_{m=0}^{\infty} \frac{\gamma}{(m+1 / 2)} \sin \left[\sigma_{m}(k)-\sigma_{m+1}(k)\right] \cos \left[\sigma_{m}(k)-\sigma_{m+1}(k)\right],
$$

using the fact that $\left[\sigma_{m}(k)-\sigma_{m+1}(k)\right]=\arctan [\gamma /(m+1 / 2)]$. The summation [32] in the above equation $(k=m v / \hbar)$ results in a non-divergent expression for the stopping power

$$
\frac{d E}{d x}=n_{0}\left(m v^{2}\right) \frac{\gamma}{k} 2 \pi \tanh (\pi \gamma)=n_{0}\left(m v^{2}\right) \frac{Z_{1} e^{2}}{m v^{2}} 2 \pi \tanh \left(\frac{\pi Z_{1} e^{2}}{\hbar v}\right) .
$$

How a regularization (screening) will change this exact quantum-result by using the above two sides with the $\sigma_{m}(k) \rightarrow \delta_{m}(k)$ substitution is a challenging question. Answers on it may contribute to our undertanding of interpretation-difficulties as well.

\section{SUMMARY AND COMMENT}

In this paper we have investigated the problem of the asymptotic behavior of the stopping force of a degenerate electron gas for fast charged projectiles. The applied method rests on scattering phase shifts in order to implement a recently proposed [7] new form. A Hulthéntype potential, with velocity-dependent screening, is used in order to derive an exact form for the leading phase shift. For charge-conjugated stable particles, i.e., for protons and antiprotons $\left(Z_{1}= \pm 1\right)$, a reduced value for the sign-dependent Barkas term is deduced. This small value is in harmony with a previous result [6] obtained within the self-consistent mean-field framework of time-dependent density-functional theory (TDDFT) applied to cluster targets. 
Very reasonable agreement with results [28] based on large-scale numerics in TDDFT with $Z_{1}=1$ for an $\mathrm{Al}$ target, is also established. However, it would be informative to see the corresponding prediction of such a numerical calculation for antiprotons as well. Indeed, for repulsive projectiles one-electron bound states can not pose an additional problem.

From the above agreements, one might conclude that the application of the mean-field concept of dynamical projectile screening is a reasonable one in order to consider the stopping power of a degenerate fermion system for swift charged projectiles. Our result obtained for the Bethe logarithm, $L_{0}=\ln \left(2 m v^{2} / \hbar \omega_{p}\right)$, takes its conventional form. However, as it is well-known [4, 34], the same asymptotic leading term comes from Pines' description of an interacting three-dimensional electron gas [35]. There, due to a canonical transformation treatment on the many-body Hamiltonian, an individual electron is screened spherically in two-body scattering and the collective mode represents a separate degree in dissipation processes. Because of such screening the sudden scattering with the swift bare projectile will result in a finite cross section and associated energy transfer.

Straightforward application [34] of Pines' modeling results in a Barkas term $L_{1} \propto \Lambda / v^{2}$ with $\Lambda=1.781 \lambda_{T F}$, where the static Thomas-Fermi $(T F)$ parameter is about $\lambda_{T F} \simeq 1 / \sqrt{r_{s}}$. In such a two-channel modeling, $L_{1}$ and $L_{2}$ have similar $\left(\propto v^{-2}\right)$ scaling in the projectile velocity. Moreover, such an $L_{1} \propto v^{-2}$ dependence [34] would fit to the experiment-based statement made at random collisional situation [2] of stopping measurement with $Z_{1}= \pm 1$. It could allow a stronger cancelation between $L_{1}$ and $L_{2}$ at positive $\gamma$ values [4] as well. Further detailed studies, by considering the sudden-character of a swift projectile in its time-dependent interaction with localized target-states of solids, are thus desirable [36]. The observable quantity, the energy loss of a heavy projectile, is well-defined classically, but the transferable knowledge on it requires treatments at the level of quantum mechanics. 


\section{Acknowledgments}

The authors are thankful to Professor Alfredo Correa for several, very useful discussions. This work was supported partly by the Spanish Ministry of Economy and Competitiveness (MINECO: Project FIS2016-76617-P).

[1] R. Peierls, Surprises in Theoretical Physics (Princeton University Press, Princeton, New Jersey, 1979), pp. 3-6.

[2] L. H. Andersen, P. Hvelplund, H. Knudsen, S. P. Moller, J. O. P. Pedersen, E. Uggerhoj, K. Elsener, and E. Morenzoni, Phys. Rev. Lett. 62, 1731 (1989).

[3] S. P. Moller, E. Uggerhoj, H. Blume, H. Knudsen, U. Mikkelsen, K. Paludan, and E. Morenzoni, Phys. Rev. A 56, 2930 (1997).

[4] J. A. Golovchenko, A. N. Goland, J. S. Rosner, C. E. Thorn, H. E. Wegner, H. Knudsen, and C. D. Moak, Phys. Rev. B 23, 957 (1981).

[5] J. Lindhard, Nucl. Instrum. Methods 132, 1 (1976).

[6] M. Quijada, A. G. Borisov, I. Nagy, R. Diez Muiño, and P. M. Echenique, Phys. Rev. A 75, $042902(2007)$.

[7] P. L. Grande, Phys. Rev. A 94, 042704 (2016).

[8] A. B. Migdal, Qualitative Methods in Quantum Theory (Benjamin, London, 1977), p. 302.

[9] M. J. Puska and R. M. Nieminen, Phys. Rev. B 27, 6121 (1983).

[10] I. Nagy, A. Arnau, P. M. Echenique, and E. Zaremba, Phys. Rev. B 40, R11983 (1989).

[11] A. Salin, A. Arnau, P. M. Echenique, and E. Zaremba, Phys. Rev. B 59, 2537 (1999).

[12] I. Nagy and M. L. Glasser, in Many-body Approaches at Different Scales, Editors: G. G. N. Angilella and C. Amovilli, (Springer, New York, 2018), pp. 133-138.

[13] S. Kreussler, C. Varelas, and W. Brandt, Phys. Rev. B 23, 82 (1981).

[14] A. Messiah, Quantum Mechanics (Elsevier, Amsterdam, 1961).

[15] G. D. Gaspari and B. Györffy, Phys. Rev. Lett. 28, 801 (1972).

[16] E. G. d'Agliano, P. Kumar, W. Schaich, and H. Suhl, Phys. Rev. B 11, 2122 (1975).

[17] L. Bönig and K. Schönhammer, Phys. Rev. B 39, 7413 (1989).

[18] J.-M. Tang and D. J. Thouless, Phys. Rev. B 58, 14179 (1998). 
[19] I. Nagy and A. Zawadowski, J. Phys.: Condens. Matter 21, 175701 (2009).

[20] L. Hulthén, Arkiv Mat. Fys. Astron. 28A, no. 5 (1942).

[21] R. Jost, Helv. Physica Acta 20, 256 (1947).

[22] R. Jost and A. Pais, Phys. Rev. 82, 840 (1951).

[23] S. Weinberg, Phys. Rev. 131, 440 (1963).

[24] L. de Ferrariis and N. R. Arista, Phys. Rev. A 29, 2145 (1984).

[25] R. Dutt, K. Chowdhury, and Y. P. Varshni, J. Phys. A: Math. Gen. 18, 1379 (1985).

[26] I. Nagy and A. Bergara, Nucl. Instrum. Methods B 115, 68 (1996).

[27] A. F. Lifshitz and N. R. Arista, Phys. Rev. A 57, 200 (1998).

[28] A. Schleife, Y. Kanai, and A. A. Correa, Phys. Rev. B 91, 014306 (2015).

[29] A. J. Cohen, P. Mori-Sánchez, and W. Yang, Science 321792 (2008).

[30] I. Nagy and I. Aldazabal, arXiv: 1810.12796 [quant-ph].

[31] A. D. Boardman, A. D. Hill, and S. Sampanthar, Phys. Rev. 160, 472 (1967).

[32] I. Nagy, Phys. Rev. B 51, 77 (1995).

[33] E. Zaremba, I. Nagy, and P. M. Echenique, Phys. Rev. B 71, 125323 (2005).

[34] I. Nagy, Phys. Rev. A 65, 014901 (2001).

[35] D. Pines, Elementary Excitations in Solids (Benjamin, New York, 1963).

[36] I. Maliyov, J. P. Crocombette, and F. Bruneval, Eur. Phys. J. B 91, 172 (2018). 